\title{
An Axiomatization of the Inner Core Using Appropriate Reduced Games
}

\author{
Geoffroy de Clippel ${ }^{1}$ \\ Chargé de Recherches FNRS. Department of Economics, Box B, Brown \\ University, Providence RI 02912, USA.
}

\begin{abstract}
I adapt a reduction process introduced by Serrano and Volij (1998) so that the reduced games of convex-valued games are convex-valued. I use the corresponding consistency property and its converse to axiomatize the inner core for games that are convex-valued, non-level and smooth.
\end{abstract}

\section{Introduction}

The consistency property can be used to axiomatize most solution concepts in game theory (see Thomson, 1996, for a survey). It requires that the restriction of a payoff vector in the solution of a game to a subset of players belongs to the solution of the reduced game. The key is to adequately define the reduced games.

Davis and Maschler (1965) introduce the first reduction process for cooperative games with transferable utility (TU). It involves some re-evaluation of the coalitional bargaining power. Let $N$ be the set of players, let $u \in \mathbb{R}^{N}$ be a potential agreement and let $S \subseteq N$ be a coalition. Then, the reduced game defined on $S$ is obtained by considering that each strict subset of $S$ may buy up the cooperation of any passive player (i.e. not in $S$ ), while $S$ itself has to buy up the cooperation of all the passive players, the utility 'price' of these players being specified by $u$.

Peleg (1986) observes that the Davis-Maschler consistency property can be used to axiomatize the core on the class of balanced TU-games. Peleg (1985)

Email address: declippel@brown.edu (Geoffroy de Clippel).

1 I thank Professors Enrico Minelli and Roberto Serrano for their comments. The usual disclaimer applies. 
extends the reduction process to games with non-transferable utility (NTU) and uses the corresponding consistency property (and its converse) to axiomatize the core on a large class of NTU-games.

One may question the asymmetric treatment of the coalitions in the DavisMaschler-Peleg reduction process. Serrano and Volij (1998, definition 3) indeed suggest that the grand coalition in the reduced games also considers the possibility of choosing a subset of passive players with whom to cooperate. They apply the related consistency property and axiomatize the core for a large class of production economies. I re-state their result on the class of NTU-games in section 3 .

It is natural in some contexts to restrict our attention to NTU games that are convex-valued. For instance, the players may agree on lotteries, and evaluate them according to the expected utility criterion. Unfortunately, the results discussed so far do not apply to this class of games, as the reduced game of a convex-valued game is not necessarily convex-valued (Peleg, 1985, section 7.6).

I convexify the Serrano-Volij reduction process. A natural interpretation is that coalitions may use lotteries to determine the set of passive players with whom to cooperate. I prove in proposition 2 that the analog of the axioms introduced by Serrano and Volij (1998) then characterize the inner core for games that are convex-valued, non-level and smooth.

The inner core is an appealing refinement of the core on the class of convexvalued games. It is obtained by applying the fictitious-transfer procedure of Shapley (1969) to the core defined for TU-games. The proof of my result relies on the fact that the inner core coincides with the set of feasible allocations that are immune to random blocking plans (Myerson, 1991, section 9.8; Qin, 1993; de Clippel and Minelli, 2004).

\section{Preliminaries}

If $N$ is a finite set, then $P(N)$ denotes the set of nonempty subsets of $N$. Let $S \in P(N)$ and let $\left(u, u^{\prime}\right)$ be a couple of vectors in $\mathbb{R}^{S}$. Then $u \leq u^{\prime}$ if $u_{i} \leq u_{i}^{\prime}$ for each $i \in S, u<u^{\prime}$ if $u \leq u^{\prime}$ and $u \neq u^{\prime}$, while $u<<u^{\prime}$ if $u_{i}<u_{i}^{\prime}$ for each $i \in S$. If $u \in \mathbb{R}^{N}$, then $u_{S}$ denotes the projection of $u$ on $\mathbb{R}^{S}$. Let $X$ be a subset of $\mathbb{R}^{S}$. Then $u$ is efficient in $X$ if $u \in X$ and there does not exists $u^{\prime} \in X$ such that $u<u^{\prime}$.

A game is a couple $(N, V)$ where $N$ is the finite set of players and $V$ is a correspondence that associates to every coalition $S \in P(N)$ a nonempty 
compact subset $V(S)$ of $\mathbb{R}^{S}$. The class of all games is denoted by $\mathcal{G}$.

A solution $\Sigma$ associates to every game $(N, V) \in \mathcal{G}$ a subset $\Sigma(N, V)$ of $V(N)$. The core for instance specifies the set of feasible utility profiles that no coalition can improve upon:

$$
C(N, V)=\left\{\sigma \in V(N) \mid \neg\left[(\exists S \in P(N))(\exists u \in V(S)): \sigma_{S}<u\right]\right\}
$$

for each $(N, V) \in \mathcal{G}$.

Serrano and Volij (1998) axiomatize the core in the context of production economies. I adapt their argument to my framework. Here are properties that one could impose on a solution $\Sigma$ defined on $\mathcal{G}$.

$\underline{\text { Axiom } 1}$ (One-Person Rationality, OPR) $\Sigma(N, V)=\arg \max _{v \in V(N)} v$, for each $(N, V) \in \mathcal{G}$ such that $\# N=1$.

Axiom 2 (Consistency, CONS) Let $(N, V) \in \mathcal{G}$, let $S \in P(N)$ and let $\sigma \in$ $\Sigma(N, V)$. Then $\left(S, V_{S, \sigma}\right) \in \mathcal{G}$ and $\sigma_{S} \in \Sigma\left(S, V_{S, \sigma}\right)$, where $\left(S, V_{S, \sigma}\right)$ is the reduced game with respect to $S$ and $\sigma$ defined as follows:

$$
V_{S, \sigma}(T):=\bigcup_{Q \in P(N \backslash S) \cup\{\emptyset\}}\left\{u \in \mathbb{R}^{T} \mid(\exists v \in V(T \cup Q)): v_{T}=u \wedge v_{Q} \geq \sigma_{Q}\right\}
$$

for each $T \in P(S)$.

Axiom 3 (Converse Consistency, CO-CONS) Let $(N, V) \in \mathcal{G}$ be a game with at least two players and let $\sigma \in V(N)$. If $\left(S, V_{S, \sigma}\right) \in \mathcal{G}$ and $\sigma_{S} \in \Sigma\left(S, V_{S, \sigma}\right)$ for each $S \in P(N) \backslash\{N\}$, then $\sigma \in \Sigma(N, V)$.

OPR requires that the solution is compatible with the maximization of individual utilities when there is only one player. Axiom 2 is the usual consistency property: the restriction of a payoff vector in the solution of a game to a subset of players must belong to the solution of the reduced game. As already discussed in the introduction, the reduction process differs from Peleg's (1985) definition only as far as the feasible set associated to the grand coalition in the reduced games is concerned: every coalition in the reduced game is now free to choose the set of passive players with whom to cooperate. It is indeed more natural to treat all the coalitions symmetrically. CO-CONS is a dual version of CONS. If the relevant projections of a feasible allocation $\sigma$ belong to the solution of the reduced games $\left(S, V_{S, \sigma}\right)$, then it belongs to the solution of the game $(N, V)$. Peleg (1985) also uses a property of converse consistency in his axiomatization of the core. There are two main differences. First, as already discussed, his reduced games differ from those defined in CONS. Second, his axiom is slightly stronger as he imposes that $\sigma \in \Sigma(N, V)$ whenever $\sigma_{S} \in \Sigma\left(S, V_{S, \sigma}\right)$ for each coalition $S$ with exactly two members.

Proposition 1 (Adapted from Serrano and Volij, 1998, Theorem 4) The core is the maximal solution to satisfy OPR and CONS on $\mathcal{G}$. It is also the minimal 
solution to satisfy $O P R$ and $C O-C O N S$ on $\mathcal{G}$. Hence it is the only solution to satisfy OPR, CONS and CO-CONS on $\mathcal{G}$.

Proof: 1 . The core obviously satisfies OPR. It also satisfies CONS and COCONS as the following arguments show.

1.a) CONS: Let $(N, V) \in \mathcal{G}$, let $S \in P(N)$ and let $\sigma \in V(N)$. If $\sigma \in C(N, V)$, then $\left(S, V_{S, \sigma}\right) \in \mathcal{G}$, as the finite union of compact sets is a compact set. On the other hand, if $\sigma_{S} \notin C\left(S, V_{S, \sigma}\right)$, then there exist $T \in P(S)$ and $u \in V_{S, \sigma}(T)$ such that $u>\sigma_{T}$. Hence $\sigma \notin C(N, V)$, as there exists $Q \in P(N \backslash S) \cup\{\emptyset\}$ and $v \in V(T \cup Q)$ such that $v \geq\left(u, \sigma_{Q}\right)>\sigma_{T \cup Q}$.

1.b) CO-CONS: Let $(N, V) \in \mathcal{G}$ be a game with at least two players and let $\sigma \in V(N)$ be such that $\left(S, V_{S, \sigma}\right) \in \mathcal{G}$ and $\sigma_{S} \in C\left(S, V_{S, \sigma}\right)$ for each $S \in$ $P(N) \backslash\{N\}$. I have to show that $\sigma \in C(N, V)$. It is sufficient to show that $\sigma$ is efficient in $V(N)$, as $V(S) \subseteq V_{S, \sigma}(S)$ and $\sigma_{S}$ is efficient in $V_{S, \sigma}(S)$ for each $S \in P(N) \backslash\{N\}$. Suppose on the contrary that there exists $u \in V(N)$ such that $u>\sigma$. Let $i \in N$ be such that $u_{i}>\sigma_{i}$. Hence $\sigma_{i} \notin C\left(\{i\}, V_{\{i\}, \sigma}\right)$ because $u_{i} \in V_{\{i\}, \sigma}(\{i\})$. This is impossible.

2. $\Sigma \subseteq C$ : Let $\Sigma$ be a solution that satisfies both OPR and CONS, let $(N, V) \in$ $\mathcal{G}$ and let $\sigma \in V(N)$. If $\sigma \notin C(N, V)$, then there exists $S \in P(N)$ and $u \in V(S)$ such that $u>\sigma_{S}$. Let $i \in S$ be such that $u_{i}>\sigma_{i}$. OPR implies that $\sigma_{i} \notin \Sigma\left(\{i\}, V_{\{i\}, \sigma}\right)$ because $u_{i} \in V_{\{i\}, \sigma}(\{i\})$. Hence $\sigma \notin \Sigma(N, V)$ by CONS.

3. $C \subseteq \Sigma$ : Let $\Sigma$ be a solution that satisfies both OPR and CO-CONS. I prove by induction on the cardinality of $N$ that $C(N, V) \subseteq \Sigma(N, V)$ for each game $(N, V) \in \mathcal{G}$. The result is obvious if there is just one player in the game, given OPR. Let $n$ be a positive integer. Suppose that I already proved that $C(N, V) \subseteq \Sigma(N, V)$ for every game $(N, V) \in \mathcal{G}$ such that $\# N \leq n$. Let $(N, V) \in \mathcal{G}$ be such that $\# N=n+1$ and let $\sigma \in C(N, V)$. By 1.a, $\left(S, V_{S, \sigma}\right) \in \mathcal{G}$ and $\sigma_{S} \in C\left(S, V_{S, \sigma}\right)$ for each $S \in P(N) \backslash\{N\}$. By the induction hypothesis, $\sigma_{S} \in \Sigma\left(S, V_{S, \sigma}\right)$ for each $S \in P(N) \backslash\{N\}$. By CO-CONS, $\sigma \in \Sigma(N, V)$.

The reasoning is simple. If the players in the reduced games can buy up the cooperation of passive players, then coalitional stability amounts to OPR. Peleg's (1985) argument is similar but less straightforward because he assumes that the members of the grand coalition buy up the cooperation of all the passive players in the reduced games. For instance, he establishes the maximality of the core by combining the individual rationality constraints in all the reduced games with two players.

Peleg $(1985,1986)$ applies his axioms to the set of games with a non-empty core. Hence his axiomatic characterizations are not completely independent of the core itself. Serrano and Volij (1998) can dispense with the non-emptiness axiom by altering the reduction process and by imposing OPR instead of 
‘individual rationality'.

The Davis-Maschler-Peleg reduction process can also be used to axiomatize the prekernel and the prenucleolus (Sobolev, 1975; Peleg, 1986; Serrano and Shimomura, 1998). It would be interesting to know whether similar results can be obtained with the Serrano-Volij reduction process. In view of Proposition 1 , every solution that satisfies CONS (and OPR) is a core selection. Hence CONS could actually be used only to axiomatize the intersection of the core with the prekernel and/or the intersection of the core with the prenucleolus.

\section{The Result}

A game $(N, V)$ is convex-valued if $V(S)$ is convex for each coalition $S \in P(N)$. Convex-valued games are the relevant models to consider when the players may agree on lotteries, and evaluate them according to the expected utility criterion. CONS is inappropriate in this context, as the reduction process does not preserve convexity. I suggest to adapt CONS by allowing the coalitions in the reduced games to use lotteries to determine the set of passive players with whom to cooperate.

I impose two regularity conditions. A game $(N, V)$ is non-level if

$$
\left[v_{i} \geq \max _{u \in V(\{i\})} u\right] \rightarrow\left[\left(\exists v^{\prime} \in V(S)\right): v_{S \backslash\{i\}}^{\prime}>>v_{S \backslash\{i\}}\right]
$$

for each $v \in V(S)$, each $i \in S$ and each coalition $S \in P(N)$ with at least two members. This is a very weak form of transferable utility: if an agreement $v$ for $S$ is individually rational for player $i$, then there exists an agreement $v^{\prime}$ for $S$ that makes all the members of $S \backslash\{i\}$ strictly better off, obviously at the expense of player $i$ if $v$ is efficient. In particular, if a vector $\lambda$ is orthogonal to $V(S)$ at an efficient utility profile that is individually rational for all the players, then $\lambda$ is strictly positive. Next, a convex set is smooth at a point of its boundary if it admits a unique supporting hyperplane at this point. A convex-valued game $(N, V)$ is smooth if $V(S)$ is smooth at each efficient utility profile, for each coalition $S$. The set of convex-valued games that are both non-level and smooth is denoted by $\mathcal{G}^{\prime}$.

I consider solutions defined on $\mathcal{G}^{\prime}$ in the remainder of the paper. The inner core is obtained by applying Shapley's (1969) fictitious-transfer procedure to the core defined on the class of games with transferable utility:

$$
I C(N, V)=\bigcup_{\lambda \in \mathbb{R}_{++}^{N}}\left\{\sigma \in V(N) \mid(\forall S \in P(N))(\forall u \in V(S)): \sum_{i \in S} \lambda_{i} u_{i} \leq \sum_{i \in S} \lambda_{i} \sigma_{i}\right\}
$$

for each $(N, V) \in \mathcal{G}^{\prime}$. It is the set of feasible utility profiles $\sigma$ for which there exists a vector of utility weights $\lambda$ such that no coalition can improve upon 
$\sigma$ even by making $\lambda$-weighted transfers of utilities between its members. By construction, the inner core is a subset of the core. The inclusion may be strict, as the following example shows.

Example 1 Let $(N, V) \in \mathcal{G}^{\prime}$ be the game defined as follows: $N:=\{1,2,3\}$, $V(\{i\})=[0,1]$ for each $i \in\{1,2,3\}, V(\{1,2\})=\left\{u \in \mathbb{R}_{+}^{2} \mid u_{1}^{2}+10 u_{2}^{2} \leq 910\right\}$, $V(\{1,3\})=V(\{2,3\})=\left\{u \in \mathbb{R}_{+}^{2} \mid u_{1}^{2}+u_{2}^{2} \leq 2\right\}$ and $V(\{1,2,3\})=\{u \in$ $\left.\mathbb{R}_{+}^{3} \mid u_{1}^{2}+u_{2}^{2}+u_{3}^{2} \leq 300\right\}$. The payoff profile $\sigma:=(10,10,10)$ belongs to the core but not to the inner core. To see that $\sigma$ does not belong to the inner core, observe that any vector of weights supporting $V(N)$ at $\sigma$ is proportional to $(1,1,1)$ and that coalition $\{1,2\}$ could improve upon $\sigma$ if its members were able to transfer utility at this rate (for instance by achieving $(30,1)$ and transferring fourteen units of utility from player 1 to player 2 ).

$\underline{\text { Axiom } 4}$ (One-Person Rationality, OPR) $\Sigma(N, V)=\arg \max _{v \in V(N)} v$, for each $(N, V) \in \mathcal{G}^{\prime}$ such that $\# N=1$.

Axiom 5 (Consistency', CONS') Let $(N, V) \in \mathcal{G}^{\prime}$, let $S \in P(N)$ and let $\sigma \in \Sigma(N, V)$. Then $\left(S, V_{S, \sigma}^{\prime}\right) \in \mathcal{G}^{\prime}$ and $\sigma_{S} \in \Sigma\left(S, V_{S, \sigma}^{\prime}\right)$, where $\left(S, V_{S, \sigma}^{\prime}\right)$ is the reduced game with respect to $S$ and $\sigma$ defined as follows: ${ }^{2}$

$$
V_{S, \sigma}^{\prime}(T):=c o\left[V_{S, \sigma}(T)\right]
$$

for each $T \in P(S)$.

Axiom 6 (Converse Consistency', CO-CONS') Let $(N, V) \in \mathcal{G}^{\prime}$ be a game with at least two players and let $\sigma \in V(N)$. If $\left(S, V_{S, \sigma}^{\prime}\right) \in \mathcal{G}^{\prime}$ and $\sigma_{S} \in \Sigma\left(S, V_{S, \sigma}^{\prime}\right)$ for each $S \in P(N) \backslash\{N\}$, then $\sigma \in \Sigma(N, V)$.

CONS' and CO-CONS' are the analog of CONS and CO-CONS obtained by convexifying the reduction process.

The inner core is the only solution to satisfy these three axioms on $\mathcal{G}^{\prime}$. I need the following lemma to prove this result. It is a convenient reformulation of previous results stating that any feasible allocation that is immune to random blocking plans belongs to the inner core (Myerson, 1991, section 9.8; Qin, 1993; de Clippel and Minelli, 2004).

Lemma 1 (Adapted from de Clippel and Minelli, 2004, Proposition 4) Let $(N, V) \in \mathcal{G}^{\prime}$ and let $\sigma \in V(N)$. If $\sigma_{S} \in C\left(S, V_{S, \sigma}^{\prime}\right)$ for each $S \in P(N)$, then $\sigma \in I C(N, V)$.

Proof: Notice that $\sigma$ is efficient in $V(N)$ since $\sigma \in C(N, V)$. Let $\lambda$ be the unique normalized vector that is orthogonal to $V(N)$ at $\sigma$. Notice also that

\footnotetext{
2 'co' denotes the convex hull operator. If $A$ is a subset of some euclidian space, then $\operatorname{co}(A)$ is the set of vectors that can be written as a convex combination of finitely many elements of $A$.
} 
$\sigma_{i} \geq \max _{v \in V(\{i\})} v$ for each $i \in N$. Hence $\lambda>>$, thanks to the first regularity condition imposed on the games in $\mathcal{G}^{\prime}$. Let $S$ be a coalition. The utility profile $\sigma_{S}$ is optimal in $V_{S, \sigma}^{\prime}(S)$, as $\sigma_{S} \in C\left(S, V_{S, \sigma}^{\prime}\right)$. Let $\lambda^{\prime}$ be a vector that is orthogonal to $V_{S, \sigma}^{\prime}(S)$ at $\sigma_{S}$. Let $U:=\left\{u \in \mathbb{R}^{S} \mid\left(u, \sigma_{N \backslash S}\right) \in V(N)\right\}$. Observe that $U \subseteq V_{S, \sigma}^{\prime}(S)$ and that $\sigma_{S} \in U$. Hence $\lambda^{\prime}$ is orthogonal to $U$ at $\sigma_{S}$. The smoothness of $V(N)$ implies that $\lambda^{\prime}$ is proportional to $\lambda_{S}$, thanks to lemma 4 in the appendix. In addition, $V(S) \subseteq V_{S, \sigma}^{\prime}(S)$. Hence $\sum_{i \in S} \lambda_{i} u_{i} \leq \sum_{i \in S} \lambda_{i} \sigma_{i}$ for each $u \in V(S)$.

Example 1 illustrates lemma 1 . The utility profile $\sigma=(10,10,10)$ belongs to core but not to the inner core. Hence there must exists a coalition $S \subsetneq N$ such that $\sigma_{S} \notin C\left(S, V_{S, \sigma}^{\prime}\right)$. Indeed, $\sigma_{\{1,2\}}$ is not optimal in $V_{\{1,2\}, \sigma}^{\prime}(\{1,2\})$, as $(30,1) \in V(\{1,2\}),(\sqrt{79}, 11) \in V_{\{1,2\}, \sigma}(\{1,2\})$ and $(10,10)<\frac{(30,1)+10(\sqrt{79}, 11)}{11}$.

Lemma 1 does not hold for convex-valued games that do not satisfy the two regularity conditions, as the following example shows.

Example 2 (Adapted from de Clippel and Minelli, 2004, Example 3) Let $\sigma=$ $(3,3,3)$ and let $(\{1,2,3\}, V)$ be the game defined as follows: $V(\{i\})=\{0\}$ for each $i \in\{1,2,3\}, V(\{1,2\})=V(\{2,3\})=\left\{u \in \mathbb{R}_{+}^{2} \mid u_{1}+9 u_{2} \leq 9\right\}$, $V(\{1,3\})=\left\{u \in \mathbb{R}_{+}^{2} \mid 9 u_{1}+u_{2} \leq 9\right\}$ and $V(\{1,2,3\})=\left\{u \in \mathbb{R}_{+}^{3} \mid u \leq(3,3,3)\right\}$. It is easy to check that $\sigma_{S} \in C\left(S, V_{S, \sigma}^{\prime}\right)$ for each $S \in P(\{1,2,3\})$. Suppose that $(3,3,3)$ belongs to the inner core of $(\{1,2,3\}, V)$. Let $\lambda$ be the associated vector of utility weights. Observe that $(9,0) \in V(\{1,2\}) \cap V(\{2,3\})$ and that $(0,9) \in V(\{1,3\})$. Hence $3 \lambda_{1}+3 \lambda_{2} \geq 9 \lambda_{1}, 3 \lambda_{2}+3 \lambda_{3} \geq 9 \lambda_{2}$ and $3 \lambda_{1}+3 \lambda_{3} \geq$ $9 \lambda_{3}$. This implies that $6\left(\lambda_{1}+\lambda_{2}+\lambda_{3}\right) \geq 9\left(\lambda_{1}+\lambda_{2}+\lambda_{3}\right)$, which is impossible.

Proposition 2 The inner core is the maximal solution to satisfy OPR and $C O N S^{\prime}$ on $\mathcal{G}^{\prime}$. It is also the minimal solution to satisfy OPR and CO-CONS' on $\mathcal{G}^{\prime}$. Hence it is the only solution to satisfy OPR, CONS' and CO-CONS' on $\mathcal{G}^{\prime}$.

Proof: 1 . The inner core obviously satisfies OPR. It also satisfies CONS' and CO-CONS' as the following argument shows.

1.a) CONS': Let $(N, V) \in \mathcal{G}^{\prime}$, let $S \in P(N)$ and let $\sigma \in I C(N, V)$. I first characterize $V_{S, \sigma}^{\prime}(T)$ for each $T \in P(S)$. Observe that the set $\left\{u \in \mathbb{R}^{T} \mid(\exists w \in\right.$ $\left.V(T \cup Q)): w_{T}=u \wedge w_{Q} \geq \sigma_{Q}\right\}$ is convex for each $Q \in P(N \backslash S) \cup\{\emptyset\}$. Hence $v \in V_{S, \sigma}^{\prime}(T)$ if and only if there exist a probability distribution $\alpha$ defined over $P(N \backslash S) \cup\{\emptyset\}$, a function $x: P(N \backslash S) \cup\{\emptyset\} \rightarrow \mathbb{R}^{T}$ and a function $w$ that associates to every coalition $Q \in P(N \backslash S) \cup\{\emptyset\}$ an element $w(Q)$ in $V(T \cup Q)$ such that $v=\sum_{Q \in P(N \backslash S) \cup\{\emptyset\}} \alpha(Q) x(Q), w_{T}(Q)=x(Q)$ and $w_{Q}(Q) \geq \sigma_{Q}$, for each $Q \in P(N \backslash S)$.

I prove now that $\left(S, V_{S, \sigma}^{\prime}\right) \in \mathcal{G}^{\prime}$. The convex hull of a compact set is a set 
that is both compact and convex. Let $T \in P(S)$ be a coalition with at least two members, let $v \in V_{S, \sigma}^{\prime}(T)$, let $(\alpha, x, w)$ be some triple associated to $v$ (see the previous paragraph) and let $i \in T$. If $v_{i} \geq \max _{u \in V_{S, \sigma}(\{i\})^{\prime}} u$, then there exists $\hat{Q} \in P(N \backslash S) \cup\{\emptyset\}$ such that $x_{i}(\hat{Q}) \geq \max _{u \in V(\{i\})} u$. Since $V$ satisfies the first regularity condition, there exists $z \in V(T \cup \hat{Q})$ such that $z_{(T \cup \hat{Q}) \backslash\{i\}}>>x_{(T \cup \hat{Q}) \backslash\{i\}}$. I obtain $x^{\prime}$ by modifying $x$ as follows: $x^{\prime}(\hat{Q}):=z_{T}$ and $x^{\prime}(Q):=x(Q)$ for every $Q \in P(N \backslash S) \cup\{\emptyset\}$ different from $\hat{Q}$. Then $v^{\prime}:=\sum_{Q \in P(N \backslash S) \cup\{\emptyset\}} \alpha(Q) x^{\prime}(Q) \in V_{S, \sigma}^{\prime}(T)$ and $v_{T \backslash\{i\}}^{\prime}>>v_{T \backslash\{i\}}$. Suppose now that $v \in \mathbb{R}^{T}$ is efficient in $V_{S, \sigma}^{\prime}(T)$ in order to check the second regularity condition. Let $Q \in P(N \backslash S) \cup\{\emptyset\}$. If $\alpha(Q)>0$, then $x(Q)$ is efficient in $\left\{u \in \mathbb{R}^{T} \mid(\exists w \in V(T \cup Q)): w_{T}=u \wedge w_{Q} \geq \sigma_{Q}\right\}$. Lemma 4 in the appendix implies that $\left\{u \in \mathbb{R}^{T} \mid(\exists w \in V(T \cup Q)): w_{T}=u \wedge w_{Q} \geq \sigma_{Q}\right\}$ is smooth at $x(Q)$. Therefore $V_{S, \sigma}^{\prime}(T)$ is smooth at $v$.

I finally prove that $\sigma_{S} \in I C\left(S, V_{S, \sigma}^{\prime}\right)$. Let $\lambda \in \mathbb{R}_{++}^{N}$ be a vector that supports $\sigma$ as an inner core allocation for $(N, V)$, let $T \in P(S)$, let $v \in V_{S, \sigma}^{\prime}(T)$ and let $(\alpha, x, w)$ be some triple associated to $v$ as before. I have: $\sum_{i \in T} \lambda_{i} v_{i}=$ $\sum_{i \in T} \lambda_{i} \sum_{Q \in P(N \backslash S) \cup\{\emptyset\}} \alpha(Q) x_{i}(Q)=\sum_{Q \in P(N \backslash S) \cup\{\emptyset\}} \alpha(Q) \sum_{i \in T} \lambda_{i} w_{i}(Q)$. Hence there exists $Q \in P(N \backslash S) \cup\{\emptyset\}$ such that $\sum_{i \in T} \lambda_{i} v_{i} \leq \sum_{i \in T} \lambda_{i} w_{i}(Q)$. As $\sigma_{Q} \leq$ $w_{Q}(Q)$ and $w(Q) \in V(T \cup Q)$, I have: $\sum_{i \in T} \lambda_{i} v_{i}+\sum_{i \in Q} \lambda_{i} \sigma_{i} \leq \sum_{i \in T \cup Q} \lambda_{i} w_{i}(Q) \leq$ $\sum_{i \in T \cup Q} \lambda_{i} \sigma_{i}$. Hence $\sum_{i \in T} \lambda_{i} v_{i} \leq \sum_{i \in T} \lambda_{i} \sigma_{i}$. So $\sigma_{S} \in I C\left(S, V_{S, \sigma}^{\prime}\right)$.

1.b) CO-CONS': Let $(N, V) \in \mathcal{G}$ be a game with at least two players and let $\sigma \in V(N)$. Suppose that $\sigma_{S} \in I C\left(S, V_{S, \sigma}^{\prime}\right)$ for each $S \in P(N) \backslash\{N\}$. Hence $\sigma_{S} \in C\left(S, V_{S, \sigma}^{\prime}\right)$ for each $S \in P(N) \backslash\{N\}$. In addition, $\sigma \in C(N, V)$, as the core satisfies CO-CONS. Lemma 1 implies that $\sigma \in I C(N, V)$.

2. $\Sigma \subseteq I C$ : Let $\Sigma$ be a solution that satisfies both OPR and CONS'. It is easy to adapt item 2 in the proof of proposition 1 to show that $\Sigma \subseteq C$. The following argument proves the stronger result $\Sigma \subseteq I C$. Let $(N, V) \in \mathcal{G}^{\prime}$ and let $\sigma \in V(N)$. Suppose that $\sigma \notin I C(N, V)$. Lemma 1 implies that there exists $S \in P(N)$ such that $\sigma_{S} \notin C\left(S, V_{S, \sigma}^{\prime}\right)$. Hence $\sigma_{S} \notin \Sigma\left(S, V_{S, \sigma}^{\prime}\right)$ and $\sigma \notin \Sigma(N, V)$ by CONS'.

3. $I C \subseteq \Sigma$ : Let $\Sigma$ be a solution that satisfies both OPR and CO-CONS'. I prove by induction on the cardinality of $N$ that $I C(N, V) \subseteq \Sigma(N, V)$ for each game $(N, V) \in \mathcal{G}^{\prime}$. The result is obvious for one-player games, given OPR. Let $n$ be a positive integer. Suppose that I already proved that $I C(N, V) \subseteq \Sigma(N, V)$ for every game $(N, V) \in \mathcal{G}^{\prime}$ with at most $n$ players. Let $(N, V) \in \mathcal{G}^{\prime}$ be a game with $n+1$ players and let $\sigma \in I C(N, V)$. By 1.a, $\left(S, V_{S, \sigma}^{\prime}\right) \in \mathcal{G}^{\prime}$ and $\sigma_{S} \in I C\left(S, V_{S, \sigma}^{\prime}\right)$ for each $S \in P(N) \backslash\{N\}$. By the induction hypothesis, $\sigma_{S} \in \Sigma\left(S, V_{S, \sigma}^{\prime}\right)$ for each $S \in P(N) \backslash\{N\}$. By CO-CONS', $\sigma \in \Sigma(N, V)$.

The axioms are independent. For each non-negative integer $k$, let $\Sigma_{k}$ be the solution defined as follows: $\Sigma_{k}(N, V):=I C(N, V)$ for each game $(N, V) \in \mathcal{G}^{\prime}$ 
with $\# N<k$ and $\Sigma_{k}(N, V)=\emptyset$ for each game $(N, V) \in \mathcal{G}^{\prime}$ with $\# N \geq k . \Sigma_{0}$ (the empty solution) satisfies both CONS' and CO-CONS', but not OPR. For each $k \geq 1, \Sigma_{k}$ satisfies both OPR and CONS', but not CO-CONS'. Finally, the core satisfies both OPR and CO-CONS', but not CONS'.

The regularity assumptions are important. The inner core does not satisfy CO-CONS' on the class of all convex-valued games. Consider the game $V$ and the payoff vector $\sigma$ defined in example 2 for instance. We have: $\sigma \notin$ $I C(N, V)$ although $\sigma_{S} \in I C\left(S, V_{S, \sigma}^{\prime}\right)$ for each coalition $S$ different from the grand coalition. Also, the inner core is not the maximal solution to satisfy OPR and CONS' on the class of convex-valued games. I refer again to the game $V$ and the payoff vector $\sigma$ defined in example 2. Consider for instance the solution $\Sigma$ that coincides with the inner core for all convex-valued games except $(\{1,2,3\}, V)$ where the solution is $\{\sigma\}$. It is easy to check that $\Sigma$ satisfies both OPR and CONS'. Yet it is larger than the inner core.

I proposed a first axiomatization of the inner core in de Clippel (2002). This previous result is not related at all to proposition 2. Indeed, it showed that the inner core is a natural extension of the core defined for TU-games to some class of convex-valued NTU-games, by adapting Aumann's (1985) axiomatization of the Shapley (1969) NTU value. The key properties were the conditional sure-thing and the conditional decreasingness axioms. They linked the solution of NTU-games with the solution of some supporting TU-games. The key properties in proposition 2 are the consistency and the converse consistency axioms. They link coalitional stability with one-person rationality. This approach leads to a full axiomatization of the inner core that does not impose on the solution to coincide with the (inner) core on the class of TU-games.

\section{References}

[1] Aumann, R. J., 1985. An Axiomatization of the Non-Transferable Utility Value. Econometrica 53, 599-612.

[2] Davis, M. and M. Maschler, 1965. The Kernel of a Cooperative Games. Naval Research Logistics Quarterly 12, 223-259.

[3] de Clippel, G., 2002. An Axiomatization of the inner core. International Journal of Game Theory 31, 563-569.

[4] de Clippel, G. and E. Minelli, 2004. Two Remarks on the Inner Core. Games and Economic Behavior, forthcoming.

[5] Myerson, R. B., 1991. Game Theory (Analysis of Conflict). Harvard University press. 
[6] Peleg, B., 1985. An Axiomatization of the Core of Cooperative Games without Side Payments. Journal of Mathematical Economics 14, 203-214.

[7] Peleg, B., 1986. On the Reduced Game Property and its Converse. International Journal of Game Theory 15, 187-200.

[8] Qin, C.-Z., 1993. The Inner-Core and the Strictly Inhibitive Set. Journal of Economic Theory 59, 96-106.

[9] Schmeidler, D., 1969. The Nucleolus of a Characteristic Function Game. SIAM Journal on Applied Mathematics 17, 1163-1170.

[10] Serrano, R. and K.-I. Shimomura, 1998. Beyond Nash Bargaining Theory: The Nash Set. Journal of Economic Theory 83, 286-307.

[11] Serrano, R. and O. Volij, 1998. Axiomatizations of Neoclassical Concepts for Economies. Journal of Mathematical Economics 30, 87-108.

[12] Shapley, L. S., 1969. Utility Comparisons and the Theory of Games. In 'La Decision', edited by G. Th. Guilbaud, CNRS, 251-263.

[13] Sobolev, A. I., 1975. The Characterization of Optimality Principles in Cooperative Games by Functional Equations. In 'Mathematical Methods in the Social Sciences', edited by N. N. Vorobiev, Academy of Sciences of the Lithuanian SSR, 6, 95-151 (in Russian).

[14] Thomson, W., 1996. Consistent Allocation Rules. Rochester Center for Economic Research (RCER) Working Paper 418. 


\section{Appendix}

Lemma 2 (Intermediate Value Theorem for Correspondences) Let $\beta$ be a real number and let $F:[a, c] \rightarrow \mathbb{R}$ be a correspondence with non-empty convex values and a compact graph. If there exists a couple $(\alpha, \gamma) \in F(a) \times F(c)$ such that $\alpha<\beta<\gamma$, then there exists $b \in[a, c]$ such that $\beta \in F(b)$.

Proof: The set $S:=\{x \in[a, c] \mid(\exists \chi \in F(x)): \chi \leq \beta\}$ is nonempty (as it contains $a$ ) and bounded above by $c$. Let $b$ be its supremum. I show that $\beta \in F(b)$. Let $\left(x_{k}\right)_{k \in \mathbb{N}}$ be a sequence in $S$ such that $x_{k} \rightarrow b$ and let $\left(\chi_{k}\right)_{k \in \mathbb{N}}$ be a sequence such that $\chi_{k} \leq \beta$ and $\chi_{k} \in F\left(x_{k}\right)$ for each $k \in \mathbb{N}$. I may assume without loss of generality (because $F$ has a compact graph) that $\chi_{k} \rightarrow \chi$ for some $\chi \in F(b)$. Notice that $\chi \leq \beta$. Let $\left(\hat{x}_{k}\right)_{k \in \mathbb{N}}$ be a sequence in $[b, c]$ such that $\hat{x}_{k} \rightarrow b$ and let $\left(\hat{\chi}_{k}\right)_{k \in \mathbb{N}}$ be a sequence such that $\hat{\chi}_{k} \geq \beta$ and $\hat{\chi}_{k} \in F\left(x_{k}\right)$ for each $k \in \mathbb{N}$. I may assume without loss of generality (because $F$ has a compact graph) that $\hat{\chi}_{k} \rightarrow \hat{\chi}$ for some $\hat{\chi} \in F(b)$. Notice that $\hat{\chi} \geq \beta$. In addition, $F(b)$ is a convex set. Hence $\beta \in F(b)$.

Let $V$ be a compact and convex subset of $\mathbb{R}^{N}$, let $i \in N$ and let $r \in \mathbb{R}$. Let $f: \mathbb{R}^{N \backslash\{i\}} \rightarrow \mathbb{R}^{N}$ be the function defined as follows: $f_{i}(\phi):=r$ and $f_{j}(\phi):=\phi_{j}$ if $j \in N \backslash\{i\}$, for each $\phi \in \mathbb{R}^{N \backslash\{i\}}$. Let $U$ be the slice of $V$ obtained by intersecting $V$ with the set of vectors in $\mathbb{R}^{N}$ whose $i$ th component equals $r$ :

$$
U:=\left\{\phi \in \mathbb{R}^{N \backslash\{i\}} \mid f(\phi) \in V\right\} .
$$

It is a convex set. The next proposition states that, under mild conditions, any vector $\lambda$ that is orthogonal to $U$ at some vector $\phi^{*}$ can be extended into a vector that is orthogonal to $V$ at $f\left(\phi^{*}\right)$.

Lemma 3 Let $\phi^{*}$ be a vector that belongs to the boundary of $U$ and let $\lambda \in \mathbb{R}^{N \backslash\{i\}} \backslash$ $\{0\}$ be a vector that is orthogonal to $U$ at $\phi^{*}$. If there exists a couple $\left(v, v^{\prime}\right) \in V \times V$ such that $v_{i}<r<v_{i}^{\prime}$, then there exists $\mu \in \mathbb{R}^{N}$ that is orthogonal to $V$ at $f\left(\phi^{*}\right)$ such that $\mu_{N \backslash\{i\}}=\lambda$.

Proof: Let $\mu: \mathbb{R} \rightarrow \mathbb{R}^{N}$ be the function defined as follows: $\mu_{i}(x):=x$ and $\mu_{j}(x):=\lambda_{j}$ for each $j \in N \backslash\{i\}$ and each $x \in \mathbb{R}$. Let $F: \mathbb{R} \rightarrow \mathbb{R}$ be the correspondence defined as follows:

$$
F(x):=\operatorname{proj}_{\mathbb{R}\{i\}}\{\chi \in V \mid \mu(x) \text { is orthogonal to } V \text { at } \chi\}
$$

for each $x \in \mathbb{R}$. Observe that $F$ has non-empty convex values. Also, I have that

$$
\sum_{j \in N} \mu_{j}(x) v_{j} \leq \sum_{j \in N} \mu_{j}(x) \chi_{j} \leq x \chi_{i}+\max _{u \in V} \sum_{j \in N \backslash\{i\}} \lambda_{j} u_{j}
$$

which implies that

$$
x\left(v_{i}-\chi_{i}\right) \leq \max _{u \in V} \sum_{j \in N \backslash\{i\}} \lambda_{j}\left(u_{j}-v_{j}\right)
$$

for each $x \in \mathbb{R}$ and each $\chi \in V$ such that $\mu(x)$ is orthogonal to $V$ at $\chi$. Hence, there exists $\underline{x} \in \mathbb{R}_{-}$and $\chi_{i} \in F(\underline{x})$ such that $\chi_{i}<r$. Similarly,

$$
x\left(v_{i}^{\prime}-\chi_{i}\right) \leq \max _{u \in V} \sum_{j \in N \backslash\{i\}} \lambda_{j}\left(u_{j}^{*}-v_{j}^{\prime}\right)
$$


for each $x \in \mathbb{R}$ and each $\chi \in V$ such that $\mu(x)$ is orthogonal to $V$ at $\chi$. Hence, there exists $\bar{x} \in \mathbb{R}_{+}$and $\chi_{i} \in F(\bar{x})$ such that $r<\chi_{i}$. In addition, the graph of the correspondence $F$ restricted to $[\underline{x}, \bar{x}]$ is compact. By lemma 2, there exists $\hat{x} \in[\underline{x}, \bar{x}]$ such that $r \in F(\hat{x})$. Hence, there exists $\hat{\phi} \in U$ such that $\mu(\hat{x})$ is orthogonal to $V$ at $f(\hat{\phi})$. This implies that $\mu(\hat{x})$ is orthogonal to $V$ at $f\left(\phi^{*}\right)$, as $\sum_{j \in N} \mu_{j}(\hat{x}) f_{j}(\hat{\phi})=$ $\mu_{i}(\hat{x}) r+\sum_{j \in N \backslash\{i\}} \lambda_{j} \hat{\phi}_{j} \leq \mu_{i}(\hat{x}) r+\sum_{j \in N \backslash\{i\}} \lambda_{j} \phi_{j}^{*}=\sum_{j \in N} \mu_{j}(\hat{x}) f_{j}\left(\phi^{*}\right)$.

Lemma 3 is not valid without the existence of a couple $\left(v, v^{\prime}\right) \in V \times V$ such that $v_{i}<r<v_{i}^{\prime}$, as the next example shows.

Example 3 The set $V:=\left\{v \in \mathbb{R}^{N} \mid \sum_{j \in N} v_{j}^{2} \leq 1\right\}$ is compact and convex. Let $i \in N$, let $r:=1$ and let $\phi^{*}:=0 \in \mathbb{R}^{S \backslash\{i\}}$. Notice that $U=\{0\}$ and so $\phi^{*} \in \partial U$. If a vector $\mu$ is orthogonal to $V$ at $f\left(\phi^{*}\right)$, then $\mu_{N \backslash\{i\}}=0$. Hence it is impossible to extend any vector $\lambda$ orthogonal to $U$ at $\phi^{*}$ into some vector $\mu$ orthogonal to $V$ at $f\left(\phi^{*}\right)$.

Lemma 4 If $u$ is efficient in $V, V$ is smooth at $u$, and there exists a couple $\left(v, v^{\prime}\right) \in$ $V \times V$ such that $v_{i}<u_{i}<v_{i}^{\prime}$, then $U$ is smooth at $u_{N \backslash\{i\}}$.

Proof: Let $\mu$ be the unique normalized vector that is orthogonal to $V$ at $u$. By lemma 3, any vector $\lambda$ that is orthogonal to $U$ at $u_{N \backslash\{i\}}$ must be proportional to $\mu_{N \backslash\{i\}}$. Hence $U$ is smooth at $u_{N \backslash\{i\}}$.

Example 3 shows that lemma 4 is not valid without the existence of a couple $\left(v, v^{\prime}\right) \in$ $V \times V$ such that $v_{i}<u_{i}<v_{i}^{\prime}$. 